\title{
LOS INICIOS DEL PSICOANÁLISIS EN PERÚ: HONORIO DELGADO Y EL COMERCIO, 1915
}

\section{THE BEGINNINGS OF PSYCHOANALYSIS IN PERU: HONORIO DELGADO AND EL COMERCIO, 1915}

Ramón León

\section{Resumen}

El $1^{\circ}$ de enero de 1915 el diario El Comercio, de Lima publicó un artículo titulado "El psicoanálisis", escrito por Honorio Delgado (1892-1969), un estudiante de la Facultad de Medicina de la Universidad Nacional Mayor de San Marcos. Con este trabajo se dio a conocer entre el gran público peruano las ideas de Sigmund Freud. La presente comunicación trata de dicho trabajo, su contenido y el contexto social en el cual apareció. Honorio Delgado, con posterioridad, sería uno de los más entusiastas difusores de las ideas de Freud, llegando a escribir una biografia del psiquiatra vienés. Sin embargo, a partir de los años 1930 se aleja del psicoanálisis y llega a convertirse en uno de sus más duros críticos.

Palabras clave: Psicoanálisis; Perú; Honorio Delgado.

\section{Abstract}

In the edition on the first day of January 1915 El Comercio, an important newspaper of Lima, published "El psicoanálisis" [Psychoanalisys], an article authored by Honorio Delgado (1892-1969), a student of the Faculty of Medicine of the San Marcos University, in Lima. With this article began the history of the Freudian doctrine in Peru. This communication presents this article, its content and the social context in which it appeared. Later, Honorio Delgado was one of the most active and enthusiastic broadcasters of Freud's ideas in Latin America, 
and the author of a biography of the father of psychoanalysis. But in the $30^{\circ}$ s he became a hard critic of this psychological theory.

Key words: Psychoanalysis; Peru; Honorio Delgado.

El psicoanálisis es una doctrina psicológica (tal vez la única) que se extiende por todo el orbe. Quizás Cuba y Corea del Norte (y alguna nación centroafricana hundida en la más absoluta miseria y descontrol social) sean los únicos países en los que no se pueda encontrar a psicoanalistas practicantes. Homayoumpur (2012) ha publicado no hace mucho un libro sobre el psicoanálisis en Irán, mediante el cual, entre muchas otras cosas de interés, tomamos conocimiento de que hay un centro formativo de psicoanalistas en ese país. En la derrumbada Unión Soviética el psicoanálisis estaba proscrito; en la renacida Rusia ha recuperado su derecho a la existencia (véase Vasilyeva, 2000, Balbuena Rivera \& Sánchez-Barranco Ruiz, 2004).

En el mundo hispanohablante es Argentina el país en el cual más profundamente ha calado el psicoanálisis. Ya hacia fines de los años sesenta, la doctrina de Freud y la terapia psicoanalitica estaban muy arraigados en la nación del Río de la Plata, de tal modo que "en Argentina y hasta el día de hoy, 'psicologia' es en buena medida, sinónimo de "psicoanálisis" (Ben Plotkin \& Visacovsky, 2008, p. 151). ${ }^{1}$ Roazen (2001, p. 339) ha señalado que "sería difícil pensar en otra nación en la cual la transformación en las ideas que Freud iniciara haya tenido un mayor impacto que en la cultura argentina"
Hoy se puede afirmar sin lugar a duda alguna que el psicoanálisis forma parte de lo que se denomina la cultura popular de ese país ${ }^{2}$.

Buenos Aires es considerada como la capital latinoamericana de la teoría que creara Freud y la cantidad de psicoanalistas, de centros de formación y de revistas y libros vinculados a esta doctrina supera largamente a la de cualquier otra urbe en Iberoamérica. Wender et al. (1995) calculaban hacia fines del siglo XX que en Argentina habían 80 mil médicos, 20 mil psicólogos, más de mil analistas miembros de la Asociación Psicoanalítica Internacional y otros mil que trabajaban de modo independiente. Ocho años después, Arbiser (2003) informó de la existencia de seis instituciones psicoanalíticas de la Asociación Psicoanalitica Internacional, tres en Buenos Aires y el resto en el interior del país, pero señaló también que "el ritmo de la demanda de análisis, que parecía inagotable en años anteriores, fue mermando en forma alarmante a partir de los años 90" (p. 175)

En julio de 1991 se celebró en Buenos Aires el XXXVII Congreso Internacional de la Asociación Psicoanalítica Internacional, el primero en llevarse a cabo en el Hemisferio Sur y en América Latina, que contó con más de tres mil participantes. Sellos como Paidós (fundada en 1945) y Amorrortu (fundada en 1967), con sede en la capital 
del Río de La Plata, han contribuido a la difusión de las ideas de Freud, así como las de otros psicoanalistas, como por ejemplo Karl Abraham (e.g. Abraham, 1961: 1973), Otto Fenichel (1966), Melanie Klein (1987-1990), Armida Aberastury (1962). Amorrortu emprendió y culminó con éxito la tarea de publicar las obras completas de Freud, cuya traducción corrió a cargo de José Luis Etcheverry (1942-2000) (Freud 1978-1992).

E1 prestigio de Argentina como plaza psicoanalitica se sustenta además en el hecho de que muchos de los psicoanalistas latinoamericanos más importantes trabajaron y residieron en Buenos Aires: Angel Garma (19041993), Marie Langer (1910-1987), Armida Aberastury (1910-1972), Arnaldo Raskovsky (1907-1995), Enrique Pichon-Riviere (1907-1977), Heinrich Racker (1910-1961).

Inclusive, un psicoanalista bonaerense, Ricardo Horacio Etchegoyen (1919-), fue el primer latinoamericano elegido como Presidente de la Asociación Psicoanalítica Internacional, en $1991^{3}$.

Sin embargo, no fue Argentina el país por el cual el psicoanálisis ingresó a América Latina; tampoco lo fue Uruguay. Este es un hecho que llama la atención, porque ambos países habian recibido en el siglo XIX una importante migración proveniente del Viejo Mundo que influyó de modo decisivo en la "europeización" de la atmósfera cultural y científica de sus respectivas capitales, Buenos Aires y Montevideo. Martin \& Lovett (1981) informan que entre 1857 y 1938 Argentina recibió 4 millones 170 mil emigrantes, Brasil 4 millones 600 mil entre 1820 y 1937 , en tanto que a Uruguay llegaron 510 mil entre 1900 y 1937.

Señalemos, además, que especialmente en Argentina se podía registrar en esa época una intensa actividad en el plano de la psicologia ${ }^{4}$. En 1891, Víctor Mercante fundó el primer laboratorio de psicología experimental en la provincia de San Juan; Horacio Piñero (1869-1919), profesor de la Facultad de Filosofia y Letras de la Universidad de Buenos Aires desde 1901, habría de fundar el segundo, que tres años después trasladaría a la mencionada Facultad (Lores Arnaiz, $2005)^{5}$.

Klappenbach (2006) señala que "la temprana psicología argentina evidenciaba su proximidad con la psicología desarrollada en Francia" (p. 80). Entre 1906 y 1908, Felix Krueger (18741948), entonces un joven psicólogo alemán que con el paso de los años llegaría a ser Presidente de la Deutsche Gesellschaft für Psychologie y el más distinguido representante de la Ganzheitspsychologie, se desempeñó como docente en la Universidad de Buenos Aires (Wolfradt, 2015), ciudad a la que llegó también Carl Jesinghaus (18861948), para ser asimismo profesor en el laboratorio de psicología experimental del Instituto Nacional del Profesorado, donde permaneció hasta 1920 (Stock, 2015).

Argentina era, además, a inicios del siglo XX una tierra de promisión, un país cuyo futuro era visto como 
signado por la riqueza y el progreso (Acemoglu \& Robinson, 2012) ${ }^{6}$.

Freud, sin embargo, no menciona en su Historia del movimiento psicoanalítico (Freud, 1968), a ningún argentino o uruguayo, ni en la primera ni en la segunda edición. Años antes de emprender la redacción de esta obra, en 1911, Freud sí se refirió a un médico chileno que él supuso que tenía ascendencia alemana, German Greve (1869-1954), haciendo mención a una ponencia suya en el Congreso Internacional Americano de Medicina e Higiene (celebrado en Buenos Aires del 10 al 25 de julio de 1910), "Sobre psicología y psicoterapia de ciertos estados angustiosos" (Freud, 1911) ${ }^{7}$.

Si Chile fue, de acuerdo con el planteamiento de Ruperthuz Honorato (2014), el país del cual provino quien primero se refirió en esta parte del mundo al psicoanálisis, chileno también fue el primer psicoanalista sudamericano formado y certificado: Fernando Allende Navarro (1890-1981), quien de retorno de Europa a su país natal, publicó su tesis, El valor de la psicoanálisis en la policlinica: una contribución a la psicología clínica" [sic], pero recién en 1925 (Allende Navarro, $1925)^{8}$.

No sería, sin embargo, Fernando Allende Navarro, quien, con todos los pergaminos que poseía, daría impulso a la difusión de las ideas de Freud en América Latina. Esa difusión no partiría ni de Santiago, ni de Buenos Aires, o Montevideo, sino de Lima y tendría como protagonista a Honorio Delgado $(1892-1969)^{9}$.
Ciudad ubicada al otro lado de los Andes, Lima había sido durante los siglos de la dominación hispana la más importante metrópoli sudamericana de habla española. Tras el proceso de independencia y de constitución de las naciones sudamericanas, fue perdiendo importancia y, tras la Guerra del Pacífico, cedió su preeminencia en la costa del Pacífico Sur a Santiago de Chile.

En el plano cultural y científico, Lima se ubicaba a inicios del siglo XX por debajo de Buenos Aires, Montevideo y Santiago. Vidal (1992) describe con las siguientes palabras a la Lima en la cual Honorio Delgado emprendió su tarea de difundir el psicoanálisis:

"Imaginemos a retrotiempo, la Lima de comienzos de siglo. Habitábanla entonces una minoria selecta, muy rica en bienes y cultura, extremosamente conservadora, y una gran masa inculta de bajo nivel económico y social. Sin duda, la tierra no estaba en ella en sazón: le faltaba el humus de la clase media, necesario para que prendiera en ella la simiente novedosa del psicoanálisis" (p. 234)

A pesar de todo esto, fue Lima la ciudad desde la cual se inició la activa difusión del psicoanálisis en el mundo hispanoamericano. Y esa tarea de difusión tiene una clara fecha de inicio: el $1^{\circ}$ de enero de 1915.

Ha pasado desapercibido entre los psicólogos y psiquiatras peruanos el centenario de la llegada del psicoanálisis al Perú, el mismo que, como diji- 
mos, ocurrió el $1^{\circ}$ de enero de 1915 , cuando un joven estudiante de medicina en la Universidad de San Marcos, todavía pocos años de recibir su título de médico, publicó en el diario $\mathrm{El}$ Comercio un artículo titulado "El psicoanálisis" (Delgado, 1915). Ese estudiante se llamaba Honorio Delgado.

En la edición primera de $\mathrm{El} \mathrm{Comer-}$ cio en el año 1915 aparecen noticias acerca de la Primera Guerra Mundial, que se extendía ya casi medio año. Y, en lo que podriamos llamar la sección de contribuciones especiales, se publicó el trabajo de Honorio Delgado. Se trata de un artículo de extensión relativamente breve, en el cual se da a conocer la doctrina de Freud y se expone en términos sucintos los principales elementos de ella. Como se comprenderá, no le era posible al autor extenderse en su exposición, algo que haría años después en su tesis de medicina, que se titularía también El psicoanálisis (Delgado, 1919).

Creemos conveniente reproducir los cuatro primeros párrafos de este artículo, en los cuales se expresa el interés de Delgado por el psicoanálisis:

"El psicoanálisis, nacido hace algo más de veinte años, de la observación de un vulgar caso de histerismo, y no teniendo en los albores de su existencia más dominio que el limitado a cierta rama de la medicina mental, es hoy un método de valor y aplicaciones universales: su extensión en la actualidad invade las esferas de todas las ciencias, y aún de la filosofia y el arte.
Para dar una idea de la producción bibliográfica de la escuela psicoanalista, diremos solo que seis revistas dedicadas únicamente a sus publicaciones no bastan para monopolizarla; y apenas si hay algún periódico de psicología o medicina cuyas columnas no hayan sido ocupadas alguna vez por trabajos de esta índole.

Pocas cuestiones han suscitado más acaloradas polémicas en las academias científicas de Austria, Alemania y Suiza; los ecuánimes tudescos, al discutir el psicoanálisis han llegado a los extremos del escándalo; habiéndose visto el caso de ser tratado de loco al autor de esta magna concepción del mecanismo del alma; concepción que tiene panoramas estupendos, sublimes, frecuentemente matizados de extravagancia, de ilogismo y hasta de vulgaridad.

A pesar de lo sencillo del fundamento primordial de la doctrina, dificil tarea es sintetizar lo que se entiende hoy por psicoanálisis; pues sus aplicaciones, como ya lo hemos anotado, son infinitas; sin embargo, encararemos, aunque brevemente, sus diferentes cuestiones fundamentales, siguiendo la historia de su desarrollo".

Delgado se revela en ese primer trabajo como alguien entusiasmado por la doctrina y la terapia psicoanalíticas. Así, escribe: 
"La pedagogía, y también la ética, beneficia del método psicoanalítico, porque permite comprender intimamente el alma del niño, con sus tendencias más ocultas. Su conocimiento enseña a no agotar las valiosas energías de los instintos, por perversos que sean; cuya represión brusca es de las más fatales consecuencias para el porvenir del educando, y que más bien transformados por sublimificación llegan a elevarse a la categoría de virtudes. La educación, basada en las adquisiciones del psicoanálisis, hará, en el porvenir, la profilaxia de la locura y el crimen"

Una lectura detenida de este primer trabajo de Delgado permite reconocer que Delgado no siempre sigue la línea ortodoxa del psicoanálisis, reclamada por Sigmund Freud sino que hay, como afirma Rey de Castro (1986), una suerte de yuxtaposición de las ideas del creador del doctrina con planteamientos jungianos ${ }^{10}$ $\mathrm{y}$ especialmente adlerianos, a pesar de que tanto Adler como Jung habian tomado ya distancia con Freud.

El artículo concluye de la siguiente manera:

"En efecto, el concepto exclusivista del libido es lo más arbitrario del psicoanálisis; es por eso que, entre sus secuaces, Freud ha visto nacer un grupo de disidentes encabezados por Adler, quien ha forjado una teoría más amplia $\mathrm{y}$, a nuestro parecer, más conforme con la realidad psicológica: todo individuo, según ella, desde la más tierna edad, en el continuo conflicto con la realidad tiende a afirmar su personalidad, imponiendo su ritmo; la acción del medio cósmico, vital y social, suscita en el mecanismo psíquico del ser, reacciones simbólicas de defensa, por las que se libra de la tensión que en él engendran las necesidades" (p. 17).

Puede percibirse en las líneas previas la simpatía de Delgado por las ideas de Adler ("quien ha forjado una teoría más amplia y, a nuestro parecer, más conforme con la realidad psicológica"), de las que se alejaría a mediados de los años $1920^{11}$.

El que en este artículo, dedicado al psicoanálisis, Delgado mencione a Jung y a Adler, cuando ambos habian dejado de ser discipulos para convertirse en disidentes de Freud, podría en principio atribuirse a la falta de información del estudioso peruano acerca de la ruptura del padre del psicoanálisis con ambos. La distancia entre Lima y Viena justificaría plenamente esa desinformación.

Sin embargo, lo cierto es que Delgado estaba bastante bien informado sobre el particular. Evidencia de ello lo constituye el hecho que el peruano menciona el artículo "Das Interesse an der Psychoanalyse", aparecido en la revista Scientia en 1913 (Freud, 1913), la misma en la cual Adler publicaría un trabajo dando a conocer su doctri- 
na (Adler, 1913). Delgado recordaría años después la lectura del trabajo de Freud, pero podemos suponer que también leyó el de Adler ${ }^{12}$.

Cuatro preguntas surgen con respecto a la publicación que comentamos: (1) ¿quién era Honorio Delgado?; (2) ¿Cómo así tomó conocimiento Delgado de esta doctrina?; (3) ¿por qué escogió El Comercio para publicar su trabajo?; y, (4) ¿cuál fue la repercusión del mismo en nuestro medio?

Respondamos a la primera pregunta. Honorio Delgado, al momento de escribir su artículo para el diario $E l$ Comercio, era un estudiante sanmarquino próximo a acabar sus estudios. Natural de Arequipa, cursó estudios secundarios en el Colegio Nacional de la Independencia de esa ciudad y, después de estudiar en la Universidad del Gran Padre San Agustín, de su ciudad natal, en la que optó el grado de bachiller en ciencias naturales en 1914 con la tesis "Las grandes cuestiones de la herencia", se trasladó a Lima para cumplir estudios de medicina en la única facultad que había en todo el país.

Ya en Lima, destacaría pronto entre los estudiantes de medicina y optaría por la especialidad psiquiátrica, que sin embargo era poco prestigiada.

Poseedor de una voluntad de hierro, de una severa disciplina en los estudios y de una gran ambición, Delgado emprendió un programa autodidacta de perfeccionamiento, que le permitió conocer varios idiomas y estar al tanto de los últimos avances de la medicina $y$, en particular, de la psiquiatría.
En 1916 se instala la psiquiatría en el Perú al crearse en la Facultad de Medicina de San Marcos la Cátedra de Enfermedades Mentales y del Sistema Nervioso, fundada por Hermilio Valdizán, de quien Delgado sería primero ayudante y después discípulo.

En 1929, a la muerte prematura de Valdizán, asume la cátedra que él había dejado vacante y la regentaría por varias décadas, al mismo tiempo que continuaba con su labor asistencial institucional (Hospital Víctor Larco Herrera) y privada y su ininterrumpida tarea publicista. En los años treinta aparece su Psicología, escrita al alimón con Mariano Iberico (Delgado \& Iberico, 1933), y en los cuarenta La personalidad y el carácter. A comienzos de la década de los 50 da a la luz su Curso de psiquiatría (Delgado, 1953).

Tanto Psicología como el Curso de psiquiatria han sido obras que han alcanzado varias ediciones, lo que habla de la amplia lectoría que ellas han tenido y de la influencia que han ejercido tanto en la psicología como en la especialidad psiquiátrica.

Ideológicamente, Delgado procede a alejarse de manera paulatina del psicoanálisis criticando numerosos aspectos de él hasta convertirse en uno de sus más acervos críticos.

Con respecto a la segunda pregunta, sabemos que Delgado ya desde su juventud era un lector voraz y que, encaminado a escoger la especialidad de la psiquiatría, trataba de mantenerse informado agenciándose la literatura más reciente sobre el particular, no 
solo nacional (muy escasa, por cierto) sino también extranjera. Para eso había emprendido el estudio de varias lenguas extranjeras, entre ellas el alemán. Muchos años después de haber publicado su trabajo en el diario limeño y ya alejado de la doctrina psicoanalítica, Delgado señaló que en la revista Scientia $^{13}$ publicada en Italia pero que llegaba a Lima, había leído a mediados de la segunda década del siglo XX trabajos de Sigmund Freud y que, a partir de esa lectura, se había interesado por las ideas del psiquiatra vienés (Delgado, 1940) ${ }^{14}$.

Debe asimismo mencionarse la influencia de Hermilio Valdizán (18851929), el maestro de Honorio Delgado. Formado como médico en la Universidad de San Marcos, Valdizán se alejó del Perú durante unos años (entre 1911 y 1914) para cumplir una etapa de perfeccionamiento profesional en Italia, en donde estableció una relación de discipulado con Sante de Sanctis (1862-1935), la más importante figura de la psiquiatría italiana de su época ${ }^{15}$.

De Sanctis trató a lo largo de su carrera académica algunos temas que también despertaron el interés del padre del psicoanálisis, especialmente el de los sueños, al que tanto el psiquiatra italiano como Freud dedicarian sendos libros (Freud, 1968; De Sanctis, 1896, 1898, 1899) ${ }^{16 .}$.

Valdizán afinó y profundizó su formación con De Sanctis y tomó (si no lo habia hecho antes) conocimiento del psicoanálisis durante sus años en Europa. De retorno al Perú probablemente trajo información muy fresca acerca del desarrollo de esta doctrina, que fue de mucho significado para Honorio Delgado.

Con respecto a la segunda pregunta, debemos señalar que en el Perú no existía ninguna revista especializada en psiquiatría. Sería precisamente Honorio Delgado quien, conjuntamente con su maestro Hermilio Valdizán (1885-1929), fundaria en 1918 la $R e$ vista de Psiquiatría y Disciplinas Cone$\operatorname{xas}^{17}$, la primera publicación periódica peruana dedicada a esa especialidad de la medicina. Demás está decir que los trabajos de Delgado en la Revista (que apareció hasta 1924) se concentraron sobre todo en temas psicoanalíticos (León, 1986).

En ausencia de una revista especializada, Delgado optó por El Comercio, ya para entonces un periódico de gran prestigio y lectura diaria obligatoria para los peruanos, en especial para aquellos que conformaban el público educado de nuestro país.

Puede llamar la atención que Delgado optara por publicar en un diario su trabajo. Sin embargo, en psicología es posible encontrar casi una tradición sobre el particular: John B. Watson, por ejemplo, publicó trabajos en revistas que no eran psiquiátricas ni psicológicas, adaptando lógicamente su lenguaje al público al cual se dirigian esas publicaciones; también lo hicieron Wilhelm Wundt y William James, como lo han anotado Viney et al. (1981).

Cueto (1989) se refiere al hecho de que no solo Delgado sino muchos otros 
intelectuales y científicos peruanos publicaran en diarios:

"Casi todos los investigadores publicaron artículos científicos no especializados en periódicos y revistas no científicas. Delgado mismo fue un experto escribiendo para el público culto. Por ejemplo, entre 1914 y 1930, Delgado publicó 62 trabajos en periódicos y revistas no académicas, mientras que solo 34 artículos en revistas especializadas. Fue algo normal para la elite científica el dirigirse al público no académico para popularizar la ciencia, narrar un experimento famoso o explicar su propio trabajo" (pp. 111-112)

¿Cuál fue por último la repercusión del trabajo de Delgado? Sería interesante llevar a cabo un estudio sobre la recepción de este artículo. Sí podemos afirmar que con "El psicoanálisis", Honorio Delgado dio inicio a su brillante carrera como autor, que se extendería hasta poco antes de su muerte, acaecida en 1969. Desde el 1ro. de enero de 1915 hasta casi los años postreros de los años 1920, Delgado fue el más importante difusor de las ideas de Freud no solo en el Perú sino en todo el mundo de habla hispana.

Fue tal el compromiso de Delgado con el psicoanálisis y tan activa su campaña de difusión, que Freud lo mencionó en la segunda edición de su Historia del movimiento psicoanalitico (Freud, 1968), señalándolo a él y a la Revista de Psiquiatria como los más activos difusores de su doctrina en el mundo de habla hispana.

Así, el psicoanálisis no solo llegó al Perú por medio de la pluma de Delgado, también llegó a todo el mundo latinoamericano ${ }^{18}$. Este es un hecho muy interesante. En lugar de que ingresara a América Latina por una urbe como Buenos Aires, que ya en ese entonces cobijaba a una importante población de origen europeo, y que era una ciudad que tenía contactos comerciales y culturales muy intensos con el Viejo Mundo, la doctrina creada por Freud ingresó a estas latitudes por una ciudad ubicada al otro lado de los Andes, Lima, en la cual los contactos con Europa -si bien por supuesto existentes- no tenían la intensidad que sí tenía Buenos Aires, y con una vida cultural y científica mucho menor que la de la urbe bonaerense.

Honorio Delgado, como hemos dicho, prosiguió por más de una década con su activa labor publicista en pro del psicoanálisis. Publicó inclusive una biografia de Freud (Delgado, 1926), al parecer la primera escrita por alguien del mundo de habla hispana. Mantuvo además correspondencia con Sigmund Freud (Rey de Castro, 1989), quien lo consideraba como un representante de importancia de su doctrina en esta parte del mundo (Freud, 1968; Schröter, 2013). En el último cuarto de la década de los años veinte es posible registrar en su obra escrita un viraje en sus intereses y un progresivo alejamiento del psicoanálisis, que con el paso de los años se convertiría en una actitud sumamente crítica hacia esa doctrina. 
El ya citado Vidal propone una explicación para el alejamiento de Delgado del psicoanálisis:

"Delgado nunca pasó de ser un mero importador, difusor y usuario trasatlántico del producto psicoanalítico. Quiero decir que jamás se analizó reglamentariamente, no formó parte activa del grupo central de adherentes al movimiento ni se ajustó a la ortodoxia que desde un principio le impuso Freud a éste. Celoso de su independencia, acostumbrado a cincelar su perfil paso a paso, mal podría avenirse Delgado a militar como acólito de un movimiento, por más científico que pareciera ser. $\mathrm{Su}$ andadura fue siempre típicamente individual, característica de un librepensador. Sin duda que tuvo ayudas y tomó prestadas ideas y concepciones del mundo ajenas, pero jamás dejó que éstas lo desviaran de su meta personal; todo cuanto leía o escuchaba lo hacía objeto de atenta observación y crítica. Es más, no sintiéndose obligado a guardar la disciplina del grupo de iniciados, Delgado estableció relaciones con algunos disidentes, sobre todo con Adler, Jung y Rank, cosa que le reprochó Freud en alguna carta. No cabe decir, pues, que Delgado le fue infiel a Freud, ni que hubo deserción o apostasía en su alejamiento. Por la correspondencia que mantuvieron entre ambos del 19 al 34 -y de la cual se conservan unas pocas muestras procedentes de Viena- se patentiza un trato respetuoso y cordial, aunque libre de los compromisos y consignas que de ordinario presiden la supeditación del discípulo al maestro. En fin, Delgado nunca dejó de ser psiquiatra para trabajar como psicoanalista; utilizó el psicoanálisis para enriquecer y vivificar el cuerpo exangüe de la psiquiatría de entonces, y lo hizo hasta donde consideró que era necesario, adecuado y oportuno" (p. 234) 19 .

El trabajo de Delgado del $1^{\circ}$ de enero de 1915 en El Comercio (el primero de muchos que él publicaría en ese diario; véase El Comercio, 1992) era un artículo de divulgación científica, dirigido al gran público ${ }^{20}$. Detalles teóricos, interrogantes, discusiones y planteamientos alternativos en torno a tal o cual aspecto de las ideas de Freud fueron obviados en aras de la brevedad y de la claridad.

Desde 1915 hasta aproximadamente poco antes de 1930 Delgado continuó difundiendo de modo activo el psicoanálisis. Como hemos dicho, en 1926 publicó la que probablemente es la primera biografia de Sigmund Freud en castellano. La Revista de Psiquiatría y Disciplinas Conexas, que dejó de publicarse en 1924, incluyó en sus páginas numerosos trabajos de él dedicados al psicoanálisis y también innumerables recensiones y comentarios de trabajos psicoanalíticos. 
Lentamente, sin embargo, Delgado se fue alejando de la doctrina psicoanalítica. Se observa una ampliación de sus intereses, incluyendo la obra de Max Scheler y de Keyserling. Ese alejamiento se pone de manifiesto ya en los años 1930: la necrología que escribe el psiquiatra peruano es abiertamente critica. Finalmente, hacia el final de su vida, encontramos a Delgado en las antipodas del pensamiento de Freud. Pero esa es ya otra historia ${ }^{21}$.

\section{Notas}

1 "Pero la difusión del psicoanálisis [en Argentina] no se limitaba a su práctica clínica. Desde principios de los años 1960, intelectuales de izquierda no vinculados a los partidos tradicionales habían encontrado en lecturas del psicoanálisis una de las tantas herramientas hermenéuticas con las que esperaban contar para analizar la compleja y elusiva realidad social y, sobre todo, politica post-peronista (o más bien "interperonista"), vivida como en una suerte de crisis permanente (aunque esto sea un oxímoron). Intelectuales tales como León Rozitchner, Oscar Masotta, Raúl Sciarreta y, en algún punto, el propio José Bleger, encontraban o intentaban encontrar en sus apropiaciones de la teoria freudiana los instrumentos analiticos necesarios para realizar análisis sociales, dando lugar a fructíferos debates entre psicoanálisis y psicoanalistas, por un lado, y ciencias y científicos sociales, por el otro" (Ben Plotkin \& Vesacovsky, 2008: 151).

2 "Se trataba de aquella área de la cultura que Beatriz Sarlo caracteriza como "saberes del pobre" y que define como "esa mezcla desprolija de discursos sobre química e ingeniería, metalurgia y electricidad, geografias exóticas y visiones que anuncian la metrópolis futura". Estos saberes, destinados a, y apropiados por, los sectores medios y medios bajos de la sociedad que se habian beneficiado de la alfabetización masiva pero que sin embargo permanecían excluidos de los espacios de alta cultura, circulaban a través de canales no académicos y constituían una "imaginación técnica" en la cual se superponían ficción y realidad. En algunos casos esta imaginación técnica renovaba viejas obsesiones -curaciones mágicas, telepatía, o la posibilidad de comunicarse con los muertos- y las expresaba en un nuevo lenguaje supuestamente legitimado por la ciencia y la técnica modernas. De esta manera se fue generando un nuevo espacio para la recepción del psicoanálisis a nivel popular, espacio que surgió en la intersección de un discurso más abierto sobre la sexualidad y la emergencia de estas nuevas formas de conocimiento semi o seudocientíficas. El psicoanálisis se ofrecía simultáneamente como una de las nuevas "tecnologías" disponibles para la renovación de las costumbres sociales, una teoría psicológica de vanguardia y como un instrumento "científico" nuevo destinado a reconceptualizar antiguos y tradicionales temas de interés, como la interpretación de los misterios de los sueños" (Ben Plotkin, 2006, p. 532).

3 Otra psicoanalista argentina, Virginia Ungar, ha sido elegida en el 2015 presidenta de la Asociación Psicoanalítica Internacional. Ella es además la primera mujer que ocupa ese cargo en toda la historia de dicha asociación.

4 Klappenbach (1994) escribe sobre el particular: "La psicologia adquiere un notable impulso en la última década del siglo y accede a estatuto universitario cuando en 1896 se crea la Facultad de Filosofia y Letras en la Universidad de Buenos Aires" (pg. 187)

5 Es interesante anotar, sin embargo, el sentido que el término psicología experimental tenía en la Argentina de aquel entonces. Sobre el particular, Klappenbach (2006) escribe: "[...] la denominación psicología experimental en la temprana psicología argentina tenía poco que ver con el concepto de psicología experimental producida en Alemania en el último cuarto del siglo XIX. Por el contrario, la denominación psicología experimental 
se relacionaba directamente con la Introducción a la medicina experimental de Claude Bernard, obra ampliamente difundida en el país y, en la misma dirección, con los estudios médico-psicológicos como los denominaban Toulouse, Vaschide y Piéron en su obra precisamente titulada Técnica de psicología experimental" (pg. 79).

6 "La realidad histórico social de la Argentina hacia 1880 marca un rumbo. Se inicia un proyecto institucional de estabilización del poder, que a través de una elite ilustrada logra colocar a nuestro país en el mundo. Se organiza la modernización económica e institucional y la generación del ochenta se lanza a conformar las bases de la República Moderna. A fines del siglo XIX, Argentina se convirtió en la más dinámica y opulenta de las repúblicas latinoamericanas. La expansión económica adquirió una escala sin precedentes. Una densa red de ferrocarriles reemplazó al viejo sistema de transporte por carretas de bueyes y mulas y sirvieron a los fines de integrar el territorio posibilitando el desarrollo de la agricultura y la ganadería. La construcción y el comercio en las ciudades se asociaron con una industria pujante y hasta el interior más tradicional encontró como beneficiarse" (Falcone, p. 12).

7 "En términos idiomáticos, se puede llegar a afirmar que el trabajo de Greve fue una de las primeras referencias en español de las ideas de Freud, en nuestro continente. Hasta hace un tiempo se lo mencionaba como la primera en Latinoamérica, sin embargo, la evidencia muestra que ya en 1899, Juliano Moreira hacía mención a Freud en sus clases de psiquiatría en la ciudad de Bahía, evidenciando la temprana atracción que provocaban los conceptos psicoanalíticos en la región" (Ruperthuz Honorato, 2014: 1855).

8 Sobre él escribe Ruperthuz Honorato: "Este médico chileno, según las referencias, fue el primer psicoanalista formado "oficialmente" que arribó al continente. Allende Navarro pasó largos años en Europa estudiando medicina en las universidades de Suiza, Bélgica y Francia. Se formó con personalidades como Constantino von Monakow, con quien se especializó en anatomía cerebral, y el mismo Hermann Rorschach. De vuelta a Chile, validó su título de médico en la Universidad de Chile con una tesis que introduce a la práctica clínica del psicoanálisis mostrando la eficacia con una serie de casos clínicos" (Ruperthuz Honorato, 2013, p. 6).

Gazmuri (1971) agrega que los estudios de medicina los llevo a cabo en Lausana y la especialización en psiquiatría en Zurich, siendo Emil Oberholzer (1883-1958) su psicoanalista didáctico y supervisor. Allende Navarro no se dedicó a la vida académica sino restringió su actividad a la práctica privada, pero influyó y formó en psicoanálisis a figuras importantes de la psiquiatría chilena como Ignacio Matte-Blanco (1908-1995) y Carlos Núñez Saavedra (1918-1981) (Jiménez, 2002).

9 También en la capital peruana se había hablado del psicoanálisis. Sebastián Lorente de Patrón (1884-1972), escribió su tesis para optar el título de médico la tesis "Las orientaciones de la psiquiatria" en 1914, en la que hace una referencia muy general al psicoanálisis (Lorente de Patrón, 1914).

Nos parece de interés transcribir lo que Lorente de Patrón escribe sobre el psicoanálisis: "El Psico-Análisis no emplea ninguno de los métodos de orden físico; debe su origen a Freud, quien a su vez lo ha deducido de un tratamiento especial empleado por Breuer en las neurosis. Convencido Freud del determinismo general de la vida psíquica, él invita a sus enfermos a hablar libremente y a decir todo aquello que les sugiere su espíritu, realizando de ese modo la exégesis de las aspiraciones del individuo. Ulteriormente se ha extendido el procedimiento a las impresiones recogidas durante el sueño y en las interpretaciones automáticas. Los alumnos de esta escuela presentan la cuestión desde un punto de vista que tiende a substituir, en mucho, los estudios anteriores de psicología psiquiátrica. Bajo las orientaciones de Freud en Viena, Jung en Zurich, Wundt en Alemania y Janet en Francia, el Psico-Análisis ha entrado de lleno en la clínica mental, constituyendo en la actualidad un poderoso factor en la investigación del mecanismo neuro-psíquico, de las fobias, obsesiones, tendencias homosexuales, demencia precoz 
de origen paralítico y de los estados delirantes" (Lorente de Patrón 1914, pp. 30-31). Tras sus estudios en San Marcos, Lorente de Patrón partió a Europa para continuar su perfeccionamiento, habiendo realizado estancias en Francia (en la Salpêtrière de Paris), Alemania, Italia, Suiza y España (Mariátegui 1972). El tema del psicoanálisis no parece haber sido vuelto a tratar por él. La relación de sus publicaciones puede obtenerse revisando Valdivia Ponce (1981).

${ }^{10}$ Efectivamente, en el artículo que venimos comentando, Delgado hace referencia a Jung tratando de la interpretación psicoanalítica del arte: "El arte aparece a la luz del psicoanálisis -y ya lo había intuido así el gran Nietzsche-, como una actividad cuya finalidad es apaciguar las necesidades no saciadas, tanto del artista que crea, cuanto del espectador o auditor que contempla. La obra de arte no es más que la cristalización o exteriorización de anhelos, más o menos velada por la observancia de reglas estéticas. Colocándose Jung en el mismo punto de vista, interpreta de igual manera la formación de las grandes instituciones sociales: el mito, la religión, la moral, el derecho, etc., no son más que "tentativas de crear compensaciones a la satisfacción insuficiente de las necesidades". Los pueblos de exigua cultura, con creencias arcaicas en poderes omnipotentes, logran por este medio controlar, siquiera en parte, la influencia aflictiva de los rigores de la realidad inclemente".

${ }^{11}$ No sorprende que Delgado mostrara inicialmente simpatías por las ideas de Alfred Adler. Este también influyó mucho en el filósofo mexicano Samuel Ramos (1897-1959) y fue leído también con mucha atención por el argentino Aníbal Ponce, quien trabajó en una síntesis de las ideas adlerianas con los planteamientos de Jean Piaget (García 2014). Sin embargo, ni Ponce ni Ramos llamarian tanto la atención de Adler como sí lo hizo Delgado, quien fue incorporado al Comité Editorial de la Internationale Zeitschrift für Individualpsychologie, la publicación oficial del movimiento adleriano, y contribuyó con un capítulo a Heilen und Bilden, una obra que Adler editaría (Delgado, 1922; véase León, 2000).

${ }^{12}$ De hecho, Delgado se revela como bastante informado. A pesar de la brevedad de su artículo y de la naturaleza periodística del mismo, se las arregla para mencionar aparte del trabajo de Freud aparecido en Scientia, Poetry and dreams (Prescott, 1912), Der Fall Gogol (Kaus, 1912) y "La Psycho-Analyse" (Janet, 1914).

${ }^{13}$ La revista Scientia, fundada en 1907, se presentaba como "Rivista di scienza. Organo internazionale di sintes scientifica. En 1910 paso a denominarse Scientia. Rivista internazionale di síntesi scientifica. Sus fundadores fueron Federico Enrigues, uno de los más importantes matemáticos italianos, Giuseppe Bruni, químico, Antonio Dionisi, médico, Andrea Giardina, y Eugenio Rignano, ingeniero y que era el codirector de la revista con Enrigues.

Scientia era una revista muy singular. En primer lugar, se caracterizaba por su apertura a las más diversas ciencias y disciplinas, razón por la cual en sus páginas aparecieron trabajos del mundo de las ciencias naturales, las matemáticas, la filosofia y las ciencias sociales. Por ese motivo aparecieron en sus páginas contribuciones de personalidades como Einstein, Fermi, Heisenberg, Russell, Poincaré, Golgi, Eddington y Fermi. A partir del ingreso de Italia a la Segunda Guerra Mundial, la revista se ve privada de las colaboradores de muchos científicos y pensadores del extranjero y pierde prestigio. En 1988 deja de aparecer definitivamente.

En las páginas de Scientia no solo publicó Freud, sino también Adler (1914), y es muy probable que Delgado conociera las ideas de este discípulo (y después disidente) de Freud a través de la misma revista.

${ }^{14}$ En "La doctrina de Freud" Delgado (1940) resume de la siguiente manera el modo en que conoció el psicoanálisis: "Reconocemos que es motivo de satisfacción para nosotros haber abrazado con entusiasmo en la juventud las ideas de Freud y haber estudiado asiduamente después su producción, así como la de sus seguidores y sus críticos. El 
hecho es que cebado nuestro espíritu por la pasión de Nietzsche -inspirador de Freudal iniciar los estudios de medicina, acogimos con extrema curiosidad las primeras nociones acerca de la psicología de los abismo del alma. Entre las lecturas iniciales recordamos el par de artículos que Freud publicara en Scientia el año 1913, cuyo efecto fue promover en nosotros la búsqueda deliberada de las fuentes. El primer libro sistemático que estudiamos en seguida fue Psychoanalysis. Its theories and practical applications, de A. A. Brill, al que siguieron las traducciones inglesas de las obras de Freud y prácticamente todas las obras existentes por entonces en los idiomas que nos eran conocidos. El aprendizaje del alemán acabó de abrirnos la perspectiva".

La revista Scientia, a la que Delgado hace referencia, en efecto publicó en el volumen 14, correspondiente al año 1913, el trabajo de Sigmund Freud "Das Interesse an der Psychoanalyse", la primera parte en el No. 21 ("Das psychologische Interesse") y la segunda en el $\mathrm{N}^{\circ} 22$ ("Ihr Interesse für die nichtpsychologische Wissenschaften").

${ }^{15}$ Italia no era en realidad una gran potencia en materia de desarrollo de la psiquiatria, pero la cercanía cultural con el Perú era mucho mayor que la que existía con Alemania, en aquel entonces el país en el cual la psiquiatria experimentaba importantes y decisivos desarrollos. De hecho, Núñez (1983) anota que "con los comienzos del siglo XX coincide un renacimiento del influjo italiano presente en el Perú desde los días iniciales de la conquista española" (pg. 10).

Mariátegui (1981), biógrafo de Valdizán, explica de la siguiente manera la elección de Italia por parte de Valdizán:

"[...] Valdizán, para su formación, no escogió Italia, y concretamente la escuela romana de Sante De Sanctis, al azar. La experiencia personal y la "sensibilizante" escuela periodística de sus años de estudiante lo llevaron al descubrimiento de la conducta humana en sus manifestaciones extremas: el hecho delictivo en toda su dramática proyección, impactó el espíritu sensible de Valdizán y lo condujo a la búsqueda de sus causas explicativas. Estaba entonces aún vigente en Italia la escuela de Cesare Lombroso, quien creía haber encontrado en un porcentaje mayoritario de criminales una forma de degeneración, una suerte de regresión atávica por factores orgánicos en formas de conducta superadas por la evolución de las especies. Valdizán fue pues a Italia -y no a Francia, entonces uno de los centros más importantes del saber psiquiátrico- en busca de un discipulo de Lombroso y Ferri, Sante de Sanctis" (Mariátegui, 1979: 40).

${ }^{16}$ Lombardo \& Foschi (2009) señalan que De Sanctis fue el único no psicoanalista italiano que dedicó mucha parte de su obra al estudio de los sueños. De Sanctis dedicó en efecto varios libros a este tema (De Sanctis, 1896, 1898, 1899).

${ }^{17}$ El término "disciplinas conexas" debe ser especificado: la revista fundada por Valdizán y Delgado incluía entre ellas, aparte del psicoanálisis, a la neuropatologia, la psicología, la educación, el folklore, la antropología, la endocrinología, la historia de la medicina, la higiene mental y la criminología.

${ }^{18} \mathrm{El}$ propio Delgado resume en los términos siguientes el proceso de difusión de las ideas de Freud, del cual él fue decisivo protagonista: "En 1915 comenzó el movimiento psicoanalítico en el Perú, y en 1919 se publicó en Lima el primer libro en español sobre la materia: El psicoanálisis. La Revista de Psiquiatría y Disciplinas Conexas, editada en Lima desde 1918 hasta 1924, fue nuestro principal órgano de ese movimiento, que también se manifestó en publicaciones nuestras en revistas de Iberoamérica y España", destacando más adelante "la prioridad del Perú, respecto del mundo iberoamericano, en aplicar y propagar sus ideas, antes de que la popularidad las vulgarizase" (Delgado, 1949).

${ }^{19}$ Del mismo parecer es Glick quien señala que el objetivo de Delgado no era ser psicoanalista sino asimilar los métodos terapéuticos freudianos en su práctica clínica (Glick, 1996).

${ }^{20}$ El valor del artículo de divulgación científica es hoy reconocido como una expresión de los procesos de democratización que caracterizan al mundo moderno. De allí que, como 
hemos dicho, el periodismo cientifico sea hoy toda una especialidad (De Semir, 2014). Recordemos, además, que en el Perú hay una larga tradición de divulgación científica de nivel. José Carlos Mariátegui (1894-1930), en Amauta, la revista que él creara, y en las conferencias que él dictaba; Oscar Miró Quesada (1884-1981, "Racso"), y en nuestros dias Tomás Unger (1930-), son nombres que deben ser mencionados en el momento en el cual se pasa revista a esa tradición.

${ }^{21}$ Delgado no fue por cierto el único entusiasta del psicoanálisis que con el paso del tiempo se alejó de esa doctrina. Algo semejante sucedió con Gordon W. Allport (1897-1967), Alexander R. Luria (1902-1977), Fritz Perls (1893-1970) y Eric Berne (1910-1970), para solo mencionar algunos de los nombres más destacados de la psicología y la psiquiatría contemporáneas. Por último, nada menos que Lev. S. Vygotsky (1896-1934), el forjador de la teoría histórico-cultural de la psicología, hoy de tanta importancia, se mostró en su juventud muy interesado por las ideas de Freud, llegando a difundirlas en la Unión Soviética de los 1920 (Miller, 1998). 


\section{BIBLIOGRAFÍA}

Aberastury, A. (1962). Teoría y técnica del psicoanálisis de niños. Buenos Aires: Paidós.

Abraham, K. (1961). Estudios sobre psicoanálisis y psiquiatría. Buenos Aires: Hormé.

Abraham, K. (1973). Contribuciones a la teoria de la libido. Buenos Aires: Paidós.

Acemoglu, D. \& Robinson, J. A. (2012). Why nations fail. The origins of power, prosperity, and poverty. New York: Crown.

Adler, A. (1914). "Die Individualpsychologie, ihre Voraussetzungen und Ergebnisse", en: Scientia, 16, 74-87.

Allende Navarro, F. (1925). El valor del psicoanálisis en la policlínica: una contribución a la psicología clínica. Santiago: Universitaria.

Arbiser, S. (2003). "Psicoanálisis en Argentina", en: Revista Uruguaya de Psicoanálisis, 97, 159-181.

Balbuena Rivera, F. \& Sánchez-Barranco Ruiz, A. (2004). "Breve historia del psicoanálisis en Rusia2, en: Revista de la Asociación Española de Neuropsiquiatría, 90, 145-164.

Ben Plotkin, M. (2006). "El psicoanálisis antes del boom", en: Biagini, H. E. \& Roig, A. A., dirs., El pensamiento alternativo en la Argentina del siglo XX (vol. 2: Obrerismo, vanguardia, justicia social, 1930-1960). Buenos Aires: Biblos, 519-542. Ben Plotkin, M. \& Visacovsky, S. E. (2008). "Los psicoanalistas y la crisis, la crisis del psicoanálisis", en: Cuadernos Lirico, 4, 149-163.

Cueto, M. (1989). Excelencia científica en la periferia. Actividades científicas e investigación biomédica en el Perú 1890-1950. Lima: GRADE.

De Sanctis, S. (1896). I sogni e il sonno nell'isterismo e nell'epilessia. Roma: Società Dante Alighieri,

De Sanctis, S. (1898). "I sogni dei neurotici e dei pazzi. Ricerche cliniche", en: Archivio di Psichiatria e Antropologia criminale, 19, 382-408.

De Sanctis, S. (1899). I sogni. Studi psicologici e clinici di un alienista. Turín: Bocca.

De Semir, V. (2014). Decir la ciencia. Divulgación y periodismo científico de Galileo a Twitter. Barcelona: Universidad de Barcelona.

Delgado, H. (1915). El psicoanálisis. El Comercio, edición del $1^{\circ}$ de enero.

Delgado, H. (1919). El psicoanálisis. Lima: Sanmartí.

Delgado, H. (1922). Unterricht in der Philosophie des Lebens, begründet in der Individualpsychologie. En: Adler, A. y Furtmüller, C., eds., Heilen und Bilden. Munich: Bergmann, 229-232.

Delgado, H. (1926). Sigmund Freud. Lima: Southwell.

Delgado, H. (1940). "La doctrina de Freud", en: Revista de Neuro-psiquiatría, 
3 (1), 9-44.

Delgado, H. (1949). "Freud a la distancia", en: Revista de Neuro-psiquiatría, 13, 117-129.

Delgado, H. (1953). Curso de psiquiatría. Lima: edición del autor.

Delgado, H. \& Iberico, M. (1933). Psicología. Lima: edición de los autores.

El Comercio (1992). Honorio Delgado en El Comercio. Lima: El Comercio.

Falcone, R. (s/f). Historia de la psicología en Argentina. Cruce de influencias europeas y carácter nacional. http://dspace.uces.edu.ar:8180/xmlui/ bitstream/handle/123456789/922/Historia_Psicol o g \% C 3 \% A D a _ e n _ Argentina_.pdf?sequence $=1$ (recuperado el 18.11.2015).

Fenichel, O. (1966). Teoría psicoanalítica de las neurosis. Buenos Aires: Paidós, 3ra. ed.

Freud, S. (1911). G. Greve, "Sobre psicología y psicoterapia de ciertos estados angustiosos", en: Zentralblatt für Psychoanalyse, 1, 594-595.

Freud, S. (1913) "Das Interesse an der Psychoanalyse (I: Das psychologische Interesse; II: Ihr Interesse für die nichtpsychologische Wissenschaften)", en: Scientia, 14, 240-250 y 369-384.

Freud, S. (1968). Historia del movimiento psicoanalitico. En: Obras completas, vol. 2, Madrid, Biblioteca Nueva.

Freud, S. (1978-1992). Obras completas. Buenos Aires: Amorrortu, 25 vols.

Gazmuri, C. (1971). La historiografía chilena (1842-1970). Santiago: Taurus (vol. 2: 1920-1970).

García, L. N. (2014). "La civilización de la psiquis: ciencia y psicología en el pensamiento de Aníbal Ponce", en: García, L. N.; Macchioli, F. A. \& Talak, A. M., Psicología, niño y familia en la Argentina 1900-1970: perspectivas históricas y cruces disciplinares. Buenos Aires: Biblos, 97-162.

Glick, Th. F. (1996). "Science in twentieth century Latin America”, en: Bethell, L., ed., Ideas and ideologies in twentieth century Latin America, Cambridge-New York. Cambridge: University Press, 287-359.

Janet, P. (1914). "La psycho-analyse. Partie 2", en: Journal de psychologie normale et pathologique, 11, 1-36 y 97-129.

Jiménez, J. P. (2002). "Chile, and psychoanalysis", en: Erwin, E., ed., The Freud encyclopedia. Theory, therapy, and culture. New York y Londres, 83-84

Kaus, O. (1912). Der Fall Gogol. Munich: Reinhardt [Serie Schriften des Vereins für freie psychoanalytische Forschung, $\mathrm{N}^{\circ} 2$ ].

Klappenbach, h. (1994). "La recepción de Wundt en la Argentina. 1907: la creación del segundo curso de psicología en la Universidad de Buenos Aires", en: Revista de Historia de la Psicología, 15,181-197.

Klappenbach, H. (2006). "Recepción de la psicología alemana y francesa en la temprana psicología argentina”, en: Mnemosine, 2 (1), 75-86.

Klein, M. (1987-1990). Obras completas. Barcelona: Paidós Ibérica (vol. 1, 1990; vol. 2, 1987, vol. 3, 1988; vol. 4, 1990). 
Homayoumpur, G. (2012). Doing psychoanalysis in Tehran. Cambridge, Mass.: MIT Press. León, R. (1986). "La Revista de Psiquiatría y Disciplinas Conexas y su significado", en: Revista de Neuro-psiquiatria, 49, 102-121.

León, R. (2000). "Los psicólogos hispanoparlantes y la teoría de Alfred Adler en la revista Internationale Zeitschrift für Individualpsychologie" (1914-1937), en: Revista Latinoamericana de Psicología, 32, 107-126

Lombardo, G. P. \& Foschi, R. (2009). "La psicofisiologia dei sogni di Sante de Sanctis. Medizina nei secoli”, en: Arte e Scienza, 21/2, 591-609.

Lorente de Patrón, S. (1914). Las orientaciones de la psiquiatría. Lima: Universidad Nacional Mayor de San Marcos, Facultad de Medicina, tesis doctoral.

Lores Arnaiz, M. del R. (2005). "Un aporte original: la concepción de la psicologia en el positivismo argentino", en: Anuario de Investigaciones (Facultad de Psicología, Universidad de Buenos Aires), XIII, 227-233.

Mariátegui, J. (1972). "Sebastián Lorente de Patrón (1884-1972)", en: Revista de Neuro-psiquiatria, 35, 84-85.

Mariátegui, J. (1981). Hermilio Valdizán. El proyecto de una psiquiatría peruana. Lima: Biblioteca de Psiquiatría Peruana.

Martin, M. R. \& Lovett, G. H. (1981). Encyclopedia of Latin American history. Westport, CT: Greenwood Press.

Miller, M. A. (1998). Freud and the Bolsheviks. Psychoanalysis in Imperial Russia and the Soviet Union. New Haven - Londres: Yale university Press.

Núñez, E. (1983). "La cultura italiana en el Perú del siglo XX", en: Mariátegui, J. C., Cartas de Italia. Lima: Amauta, 6ta. edición, 9-34.

Prescott, F. C. (1912). "Poetry and dreams", en: Journal of Abnormal Psychology, 7, 17-46 y 104-143.

Ramos, S. (2001). El perfil del hombre y la cultura en México. México DF: Editorial Planeta Mexicana (36. reimpresión).

Rey de Castro, A. (1989). "Las cartas de Sigmund Freud a Honorio Delgado", en: Delgado, H., Freud y el psicoanálisis. Escritos y testimonio. Lima: Universidad Peruana Cayetano Heredia, 507-569.

Rey de Castro, A. (1986). "El psicoanálisis en el Perú: notas marginales", en: Debates en Sociología, 11, 229-310.

Roazen, P. (2001). The historiography of psychoanalysis. New Brunswick, New Jersey: Transaction Publisshers.

Ruperthuz Honorato, M. (2013). "El retorno de lo reprimido": el papel de la sexualidad en la recepción del psicoanálisis en el círculo médico chileno, 19101940, en: História, Ciencias, Saúde - Manguinhos, Rio de Janeiro.

Ruperthuz Honorato, M. (2014). "Germán Greve Schlegel y la recepción del psicoanálisis en Chile: la historia de un médico chileno "probablemente alemán"', en: Universitas Psychologica, 13 (5), 1847-1867.

Schröter, M. (2013). Briefe. En: Lohmann, H.-M. \& Pfeiffer, J., eds., Freud 
Handbuch. Leben - Werk - Wirkung. Stuttgart- Weimar: J. B. Metzler, 220-231.

Stock, A. (2015). Jesinghaus, Carl. En: Wolfradt, U.; Billmann-Mahecha, E. \& Stock, A., eds. Deutschsprachige Psychologinnen und Psychologen 19331945. Ein Personenlexikon, ergänzt um einen Text von Erich Stern. Wiesbaden: springer, 218-220.

Taiana, C. (2005). "Conceptual resistance in the disciplines of the mind: the Leipzig-Buenos Aires connection at the beginning of the20th century", en: History of Psychology, 8, 383-402.

Vasilyeva, N. (2000). "Psychoanalysis in Russia: the past, the present, and the future", en: American Imago, 57, 5-24.

Valdivia Ponce, O. (1981). Bibliografía psiquiátrica peruana. Lima: edición del autor.

Vidal, G. (1992). "Honorio Delgado y el psicoanálisis", en: Revista de Neuropsiquiatría, 55, 229-236.

Viney, W.; Michaels, T. \& Ganong , A. (1981). "A note on the history of psychology in magazines", en: Journal of the History of the Behavioral Sciences, 17 (2), 270-272.

Wender, L.; Torres, D. \& Vidal, I. (1995). Argentina. En: Kutter, P., ed., Psychoanalysis international. A guide to psychoanalysis throughout the world (vol. 2: America, Asia, Australia, Further European Countries), Stuttgart-Bad Cannstatt, Frommann-Holzboog, 1-26.

Wolfradt, U. (2015). Krueger, Felix. En: Wolfradt, U.; Billmann-Mahecha, E. \& Stock, A., eds. Deutschsprachige Psychologinnen und Psychologen 19331945. Ein Personenlexikon, ergänzt um einen Text von Erich Stern, Wiesbaden, springer, 262-263. 